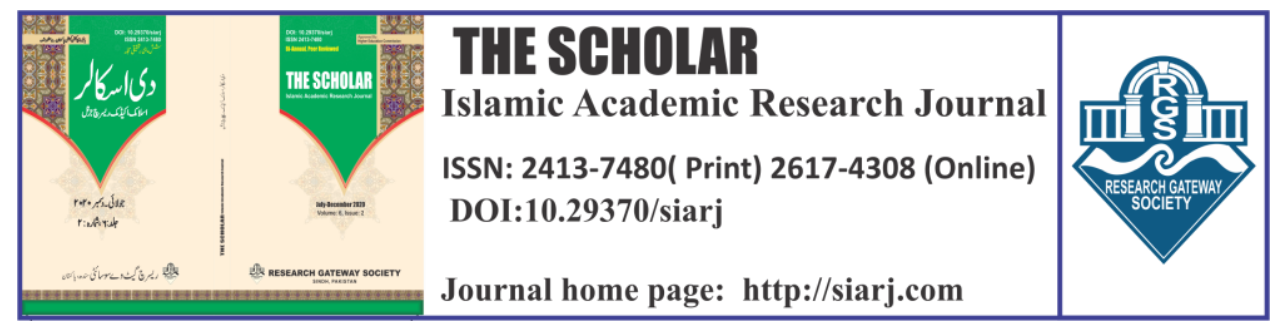

$$
\text { أثر الطب النبوي في التوعية الصحية - وباء كورونا أنموذجاً }
$$

\title{
THE EFFECT OF PROPHETIC MEDICINE ON HEALTH AWARENESS: CORONA EPIDEMIC AS A MODEL
}

\section{1- Masood Ahmad}

Assistant professor, International Islamic

University, Islamabad, Pakistan

Email : masood.ahmed@iiu.edu.pk

\section{ORCID ID:}

\section{https://orcid.org/0000-0002-9804-8314}

\section{Bashir Bahar Gadam}

Assistant professor, International Islamic

University, Islamabad, Pakistan

Email: drgadam69@gmail.com

\section{ORCID ID:}

\section{https://orcid.org/0000-0002-0998-2835}

To cite this article:

Ahmad, Masood, and Bashir Bahar Gadam. "ARABIC- THE EFFECT OF PROPHETIC MEDICINE ON HEALTH AWARENESS: CORONA EPIDEMIC AS A MODEL." The ScholarIslamic Academic Research Journal 6, No. 2 (December 27, 2020): 21-35.

To link to this article: https://doi.org/10.29370/siarj/ issue11arabic2

Journal

Publisher

DOI:

URL:

License:

Journal homepage

Published online:
The Scholar Islamic Academic Research Journal Vol. 6, No. 1 || Janurary -June 2020 || P. 21-35 Research Gateway Society 10.29370/siarj/issue 10ar7 https://doi.org/10.29370/siarj/ issue11 arabic2 Copyright c 2017 NC-SA 4.0 www.siari.com 2020-12-27

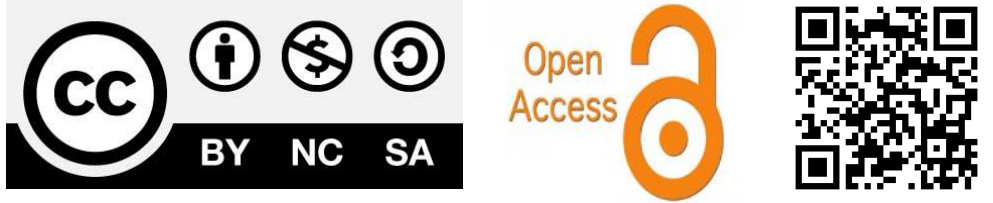


THE SCHOLAR (July -December 2020)

\title{
أثر الطب النبوي في التوعية الصحية - وباء كورونا أنوذجاً \\ THE EFFECT OF PROPHETIC MEDICINE ON HEALTH AWARENESS: CORONA EPIDEMIC AS A MODEL
}

\author{
Masood Ahmad, Bashir Bahar Gadam
}

\begin{abstract}
The modern world today, the Covid-19 Corona epidemic is sweeping the entire world, as people on earth have become prisoners of diseases that kill them without discrimination, gender or color, as it affects the ruler, princes and kings - the rich and the poor - and it has greatly damaged the social and health environment until it is no longer. People meet as they once were. Rather, people are fleeing from each other, especially sick people or those accused of this deadly epidemic as fleeing from the hungry lion or hating it as they hate meeting the enemy. The Corona epidemic blocked the wide horizon, closed commercial activities, and paralyzed life, as it became difficult for people to move from place to place completely freely. Rather, the military is from that, as governments around the world have been keen to emphasize the movement of citizens, and to close markets, shops, restaurants, and hotels, and have gone further than that by preventing public gatherings in addition to the gathering of more than five individuals in one place. In the first cycle of the spread of Corona disease, governments sought to close airports and prevent travel between cities in the same country, in addition to travel between different countries. This increased isolation between countries. From what seriously affected the economic activities. Some countries did not pay attention to this ban and did not take it seriously at first, which
\end{abstract}


increased the spread and transmission of infection from sick people to healthy people.

KEYWORDS: Effect, Prophetic Medicine, Health Awareness, Corona Epidemic

الكلمات المفتاحية: أثر، الطب النبوي، التوعية، الصحية، وباء كورونا

المدخل: - n

يجتاح العالم المعاصربأسره اليوم وباء كورونا Covid-19، حيث صار الناس على وجه الأرض أسرى الأمراض التي تفتك بهم من غير تميز ولا اعتبار لجنس ولا لون، فهو يصيب الحاكم والأمراء والملوك - أغنياء الناس وفقراءهم- وقد أضر كثيرا بالبيئة الاجتماعية والصحية حتى ما عاد الناس يلتقون كما كانوا من ذي قبل. بل صار الناس يفرون من

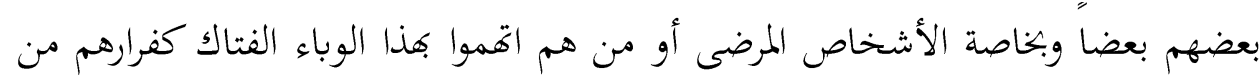

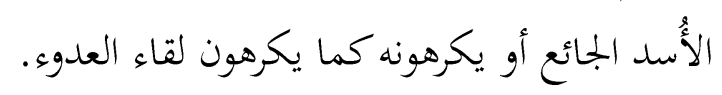
وباء كورونا سد شسع الأفق وأغلق الأنشطة التجارية، وشل حركة الحياة، حيث صار من الصعوبة بمكان أن يتنقل الناس من مكان إلى مكان بحرية تامة. بل على العكس من ذلك حيث حرصت الحكومات حول العالم على التشديد على حركة المواطنين، واغلاق الأسواق، والمحلات التجارية، والمطاعم، والفنادق، وذهبت أكثر من ذلك بمنع التجماعات العامة علاوة على بتمع أكثر من خمسة أفراد في مكان واحد. في الدورة الأولى لانتشار مرض كورونا سعت الحكومات إلى اغلاق المطارات، ومنع الأسفار بين المدن في الدولة الواحدة، إضافة إلى الترحال بين الدول المختلفة. وهو ما زاد من حدة العزلة بين الدول. مما تضررت منه الأنشطة الاقتصادية أيما ضرر. بعض الدول لم تنتبه إلى هذا الحظر ولم تتعامل معه بصورة جادة بادي الأمر وهو ما عمل على زيادة انتشار ونقل العدوى من الأشخاص المرضى إلى الأصحاء.

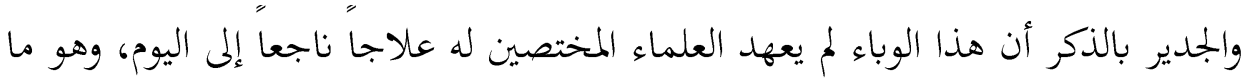
عمل على تعزيز قدرة وباء كورونا في العمل على تمديد رقعته في الإصابة بالمرض، وتوطنه 
في دول عدة حول العالم. وساعد في ذلك أن بعض الحكومات مثل حكومة دولة البرازيل

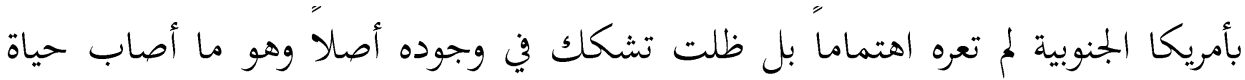

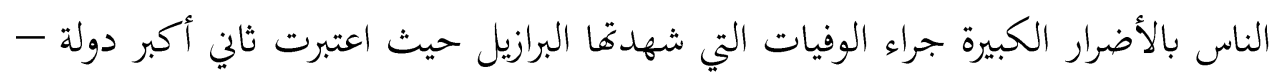

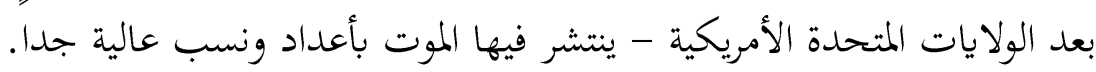

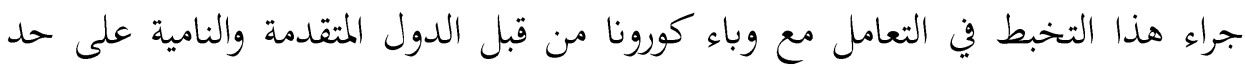

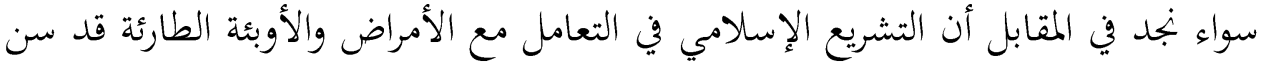

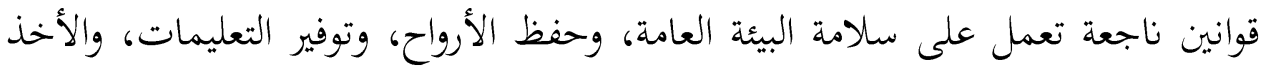

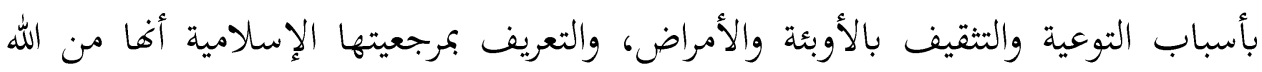

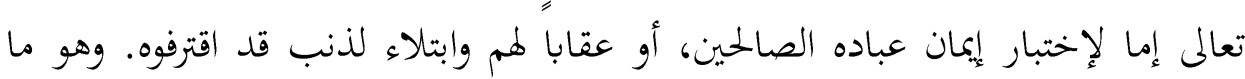

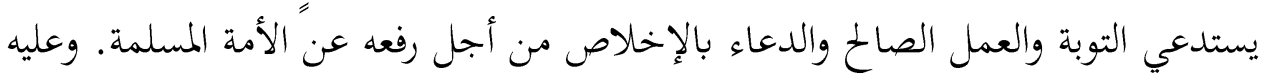

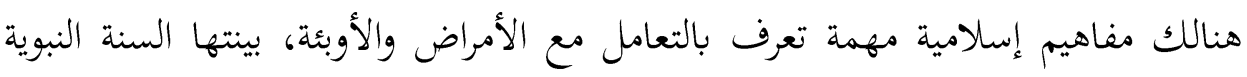

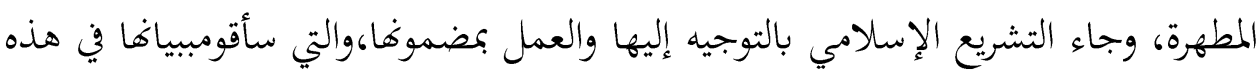
الورقة العملية المسماة: أثر الطب النبوي في التوعية الصحية- وباء كورونا أنموذجاً.

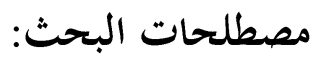

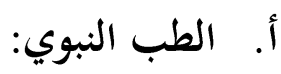

الطب النبوي هو عبارة عن مصطلح يطلق على بجموعة من النصائح المأخوذة من أحاديث البي - -

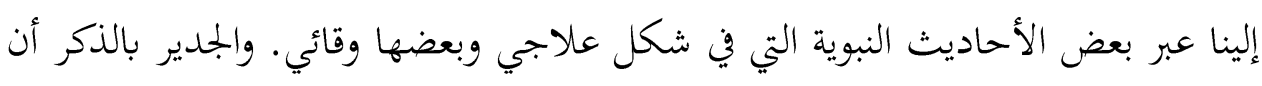

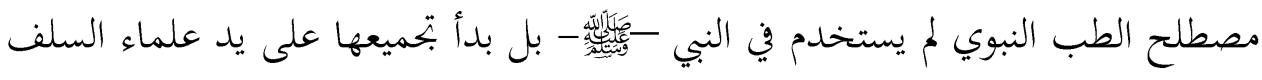

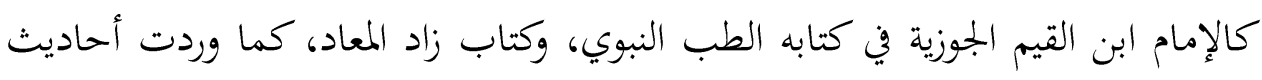

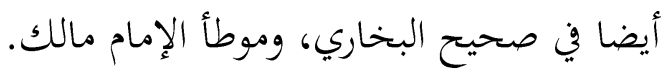
ب. الأثر: 
التأثير هو نفوذّ على إحداث أثر قوي، ويقال فلان ذو تأثير كبير، أو وافق تحت

تأثير والده، والمراد منه هنا ما تحدثه الأحاديث النبوية المتعلقة بالطب والصحة العامة من أثر في سلوك الفرد المسلم.

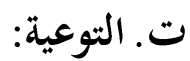

التوعية: مصدر وعي، وتوعية الناس من أسس المواطنة، وهو ما يعني جعلهم يدركون حقائق الأمور، والتوعية الصحية لها طرق عديدة تصب في هاياتحا إلى تحصين البيئة والأفراد، والجماعات من الإصابة بالأمراض أو الأوبئة الفتاكة.

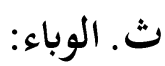

الوباء كل مرض شديد العدوى، وسريع الانتشار من مكان إلى مكان، يصيب الإنسان والحيوان والنبات، وء ادة ما يكون قاتلا كالطاعون ووباء الكوليرا، والطاعون، وكما هو

$$
\text { مفهوم الطب النبوي: من مرض كورونا. }
$$

الطب النبوي هو مصطلح إسلامي حديث نسبتهالى الأحاديث النبوية الشريفة

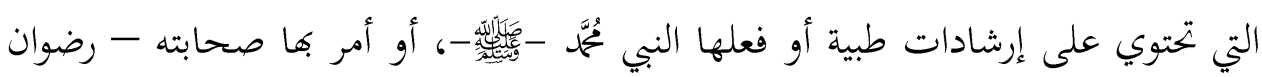
الله عليهم - في مناسبة عدة ومختلفة. وهي تشى بعظمة التشريع الإسلامي الذي جمع بين الدين والدنيا، والتي منها الحفاظ على الضرورات الخمس والتي منها الحفظ على النفس البشرية، وحمايته من القتل بغير حق، أو تعريضها للضر البدني أو الصحي أو العقلي، وقد أمر الله تعالى نبيه عند بدء الوحي بالحفاظ على صحة بدنه فقال عز وجل: "ياأيها المدثر، قم فأنذر، وربك فكبر، وثيابك فطهر، والرجز فاهجر."1 وقوله تعالى: "ولا تلقوا بأيديكم

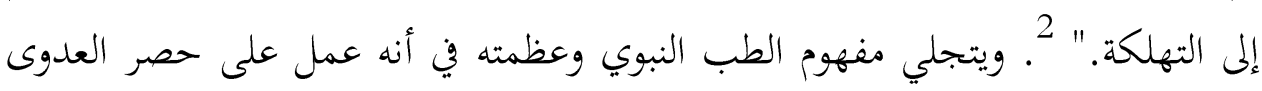

1. Al-Quran(74)-1-5

2. Al-Quran(2)-195 


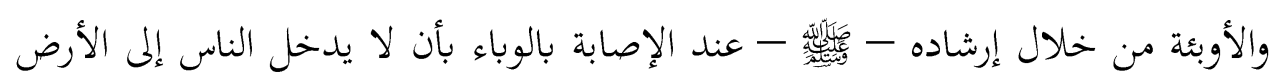

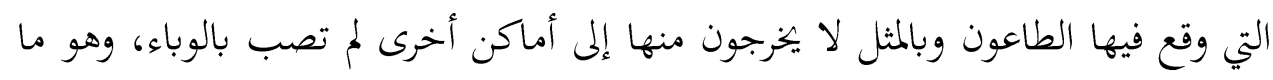
يعني حصر المرض، وتطبيق النظرية الطبية الحديثة في الامتثال إلى قواعد التباعد المكاني، والجسدي، المعمول به الآن كحلٍ ناجعِ لحصر انتشار وباء كورونا. وتتجلي أيضا أهمية الطب النبوي في أنه يأمر المسلم بالتداوي عند الإصابة بالأمراض، كما ويكرم ويبرم ممارسة الطب من غير علم ولا خبرة كافية، وألقى المسؤولية على الطبيب الذي يداوي الناس عند

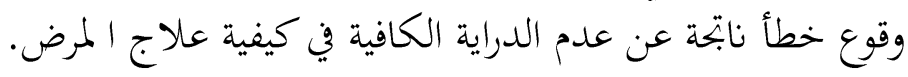

مفهوم الوباء من الناحية الإسلامية:

يعتبر المرض مهما كان نوعه ابتلاء من الله تعالى للعبد المؤمن، فهو كفارة للذنوب، وكما يثاب المبتلى به الأجر الكبير والثواب العظيم الذي يتمثل في نيل الشهادة

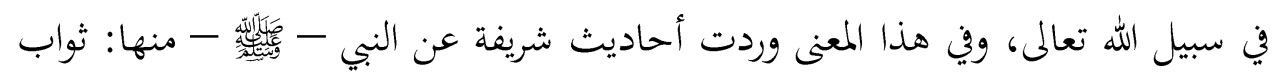

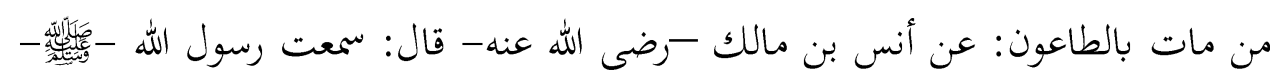

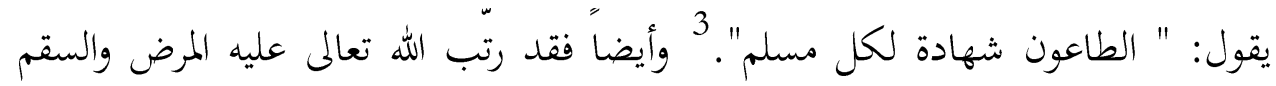
الثواب الجزيل، قد ورد عن ابن مسعود -رضى الله عنه- أن رسول الله -صلى الله عليه وسلم- قال: " ما من مسلم يصيبه أذى من مرض فما سواه إلا حط الله به سيئاته كما تشظ الشجرة ورقها" مقاصد التشريع الإسلامي:

3. Imam Muslim, Sahih Muslim, thqiq: nazar bin muhamad alfariabi, Diar tayiba, 1st edition, No.3652, (5674), 1st volume.

4 Imam al-Bukhari, Sahih al-Bukhari, Dar altaasil,Markaz albihuth wataqniat almaelumat, 1st edition,1st volume, 


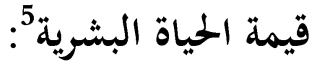

يتضح مقصد التشريع الإسلامي في مفهوم الوباء العام (كالطاعون) على حصر العدوى عن من ابتلى بها وأن لا تتعدى إلى غيره. وبالمثل توجيه الأصحاء إلى عدم مخالطة أو التواجد في البيئة التي أصيبت بالوباء. وهذا المفهوم هو ما أكد عليه الطب الحديث بخاصة في حالة كورونا-19. حيث عمدت منظمة الصحة العالمية اتباع نفس التوجيهات النبوية الخاصة بسلامة المرضى، وعدم نقل العدوى إلى مصابين آخرين، كما أرشدت على مسألة التبعاد الجسدي والمكاني. Physical distance، و مقصد التشريع الإسلامي في ذلك يكمن في تعظيم النفس البشرية والتأكيد على قيمة الحياة، وعدم إزهاق النفس

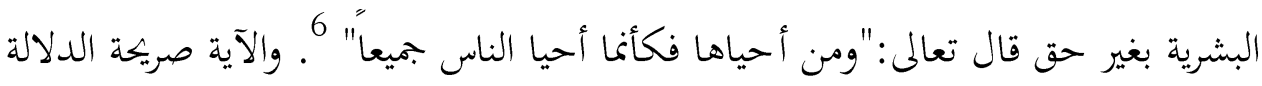

$$
\text { بالحث على الحفاظ على النفس التي بين جناحينا. }
$$

المقصد الثاني من مقاصد التشريع الإسلامي الذي أشار إليه الطب النبوي وأثره في صحة الفرد جاءت نصوصه توضح مبدأ عدم الضرر ومن ذلك قوله تعالى " إن الله يحب التوابين

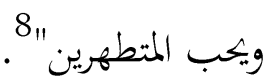

5. Al-Tahir Ibn Ashoor - Objectives of Islamic Law - Investigator: Muhammad Al-Habib Ibn Al-Khawja - Publisher: Ministry of Endowments and Islamic Affairs, Qatar - 2004 V. 2 P 139).

6. Al-Quran (32)-5

7. Ibne Ragab - The golden rule in Islamic dealings - Investigator: Ihab Hamdi Ghaith - Publisher: Dar Al-Kitab Al-Arabi - First Edition, 1990, P. 15

${ }^{8}$. Al-Quran (222)-2 


\section{أثر الطب النبوي في التوعية الصحية - وباء كورونا أنموذجاً}

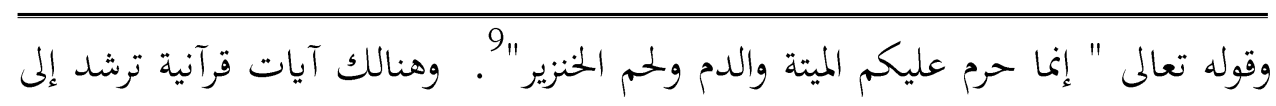

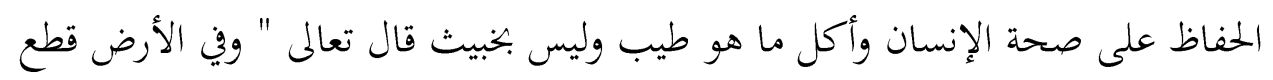

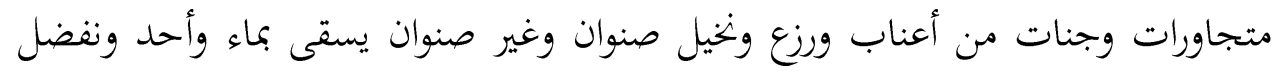
بعضها على بعض في الأكل إن في ذلك لآيات لقوم يعقلون." 10 . وبالمثل هنالك أحاديث نبوية تأمر بالحفاظ على صحة الإنسان منها قول النبي -صلى لـولى الله

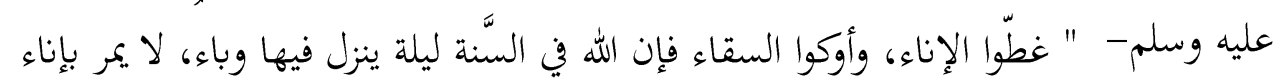

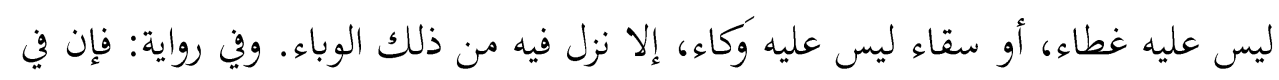

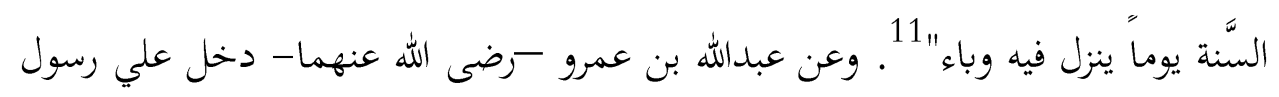

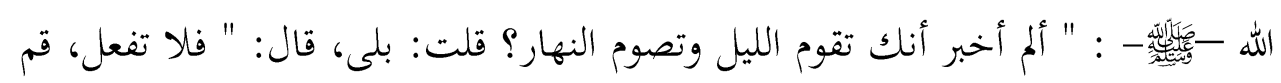

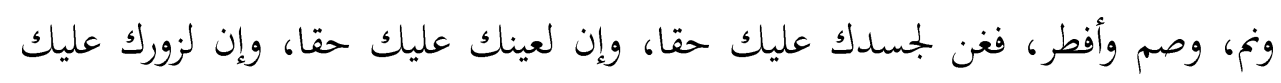

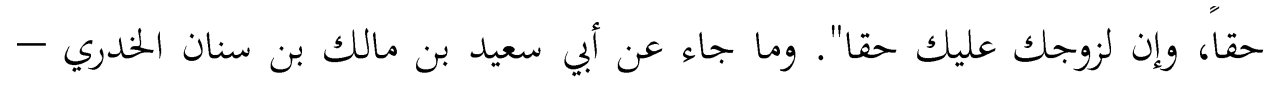

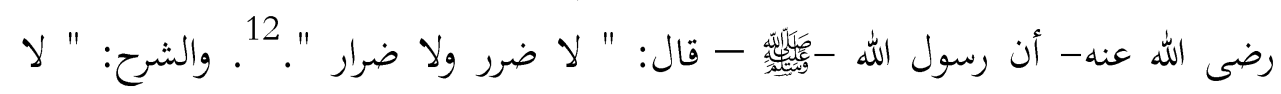

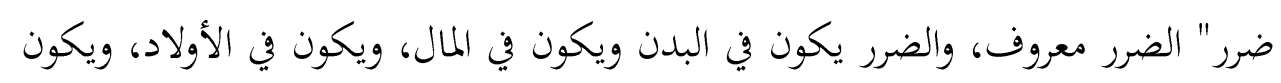

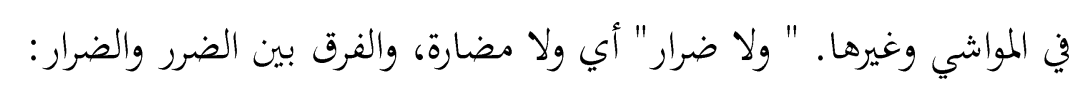

-

ذلك: رجل له جار وعنده شجرة يسقيها كل يوم، وإذا بالماء يدخل على جاره

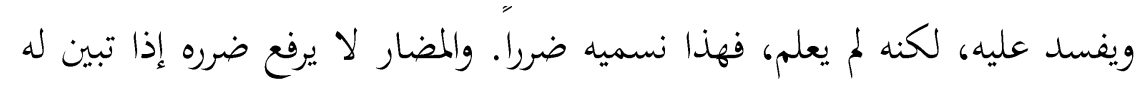

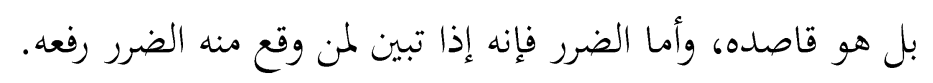

\footnotetext{
${ }^{9}$ Al-Quran (173)-2

${ }^{10}$. Al-Quran(4)-13

11. Imam Muslim, Sahih Muslim, Hadith No. (3870), Kitab Al-a'shariba.

12. Imam Ibn Majah, Sunan ibn Majah, muhamad nasir aldiyn al'albani, maktabat almaearif, $1^{\text {st }}$ edition,volume No.2, Hadith No. (2321).
} 
أثر الطب النبوي في التوعية الصحية - وباء كورونا أنموذجا

- فالقاعدة: متى ثبت الضرر وجب رفعه، ومتى ثبت الإضرار وجب رفعه مع عقوبة

قاصد الإضرار. وهذه الحديث يعتبر قاعدة من قواعد الشريعة، وهي أن الشريعة

لا تقرٌ الضرر، وتنكر الإضرار أشد وأشد.

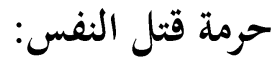

ومن مقاصد التشريع الإسلامي في حفظ النفس وتبشيع جريمة قتلها قوله تعالى: " من أجل ذلك كتبنا على بني إسرائيل أنه من قتل نفسا بغير نفس أو فساد في الأرض فكأنما

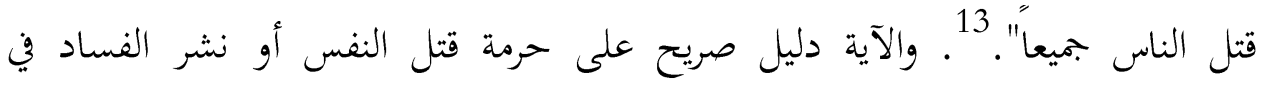
الأرض. تضمنت الآية النهي عن العبث بالبيئة التي تعمل على جلب الأوباء الخطيرة ونشرها بين الناس. وقد باتت مسألة صناعة الفيروسات في المعامل الطبية وتسيسها لإلحاق الضرر باقتصاد الدول، وسلامة البشرية ونشر الرعب والأمراض الفتاكة فيها من المحرمات بمكان. وبما أفهلا يصنف الطب النبوي ضمن علوم الطب أو بديل عن الاستعانة بالأطباء، فقد حث

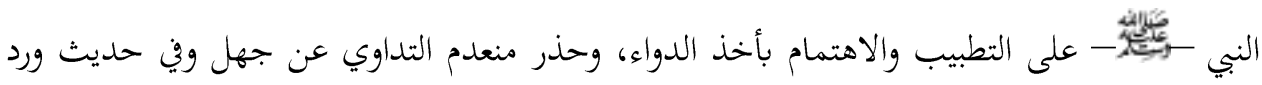

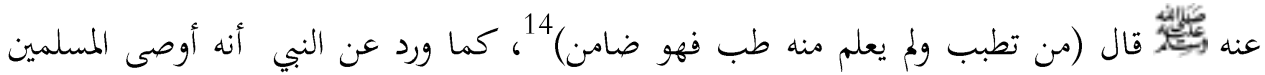

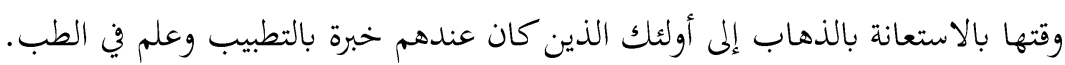

\section{دور الإرشاد الديني في التوعية الصحية:}

يعتبر الإرشاد الديني من أهم العوامل التوعوية في إصلاح وتوجيه الفرد إلى الصلاح والسلوك القويم، حيث إن تعاليم الشريعة الإسلامية السمحة قد تركزتفي الحثث على الأخذ بالحيطة والحذر، لذا جاءت أحاديث تأمر بالأستعاذة من الأسقام. وسؤال الله من الأمراض المستعصية والتعوذ به من شر الحاسدين والكائدين، وفعل ذلك في المأكل

13. Al-Quran (32)-5

14. Imam Abu Dawood,Sunan Abu Dawood, muhamad nasir aldiyn al'albani, maktabat almaearif, $1^{\text {st }}$ edition, volume No.1, Hadith No. (513). 


\section{أثر الطب النبوي في التوعية الصحية - ـوباء كورونا أنموذجاً}

والمشرب، وقد وردت معاني الأيات والأحاديث تقر هذا المبدأ بأن الله طيب ولا يقبل إلا طيبا. بل أمرت الآيات بالأكل من الطيب والابتعاد عن الخبيث. ولا شك أخهينغي على القائمين على المؤسسات الصحية، والمراكز الطبية، والعاملين في الصحة العامة، أن يجتهدوا في التوعية الصحية من أجل العمل بجد في الاستفادة من جهود المؤسسات الدينية الرسمية أو المنظمات الإسلامية الطوعية، وذلك باستخدام منابر التوعية العديدة التي تمتاز بها المؤسسات الإسلامية في توعية المجتمع وتثقيف شبابه لعدم الوقوع في براثين الأوبة والمخدرات التي تعمل على ضعف الأجسام وبتعلها عرضة للأمراض.

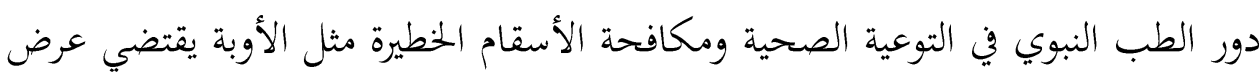
أدلة الشريعة السمحة التي فيها التحذير الشديد من الاضرار بالبيئة في الأخذ بمبدأ لا ضرر ولا ضرار. وحرمة شرب الخمر، وما نص القرآن الكريم وورد في الأحاديث النبوية من العقاب والوعيد لأولئك المخالفين لأوامر الله تعالى. لا شكأن الأحاديث النبوية لها أثر مهم في تثمين مبدأ التوعية الصحية العامة والخاصة والتي تعمل على حصر الأمراض وعدم مخالطة أصحابها (15). ويكمن دور وسائل الإعلام في مكافحة الأوبة وذلك تعزيز دور التثقيف الصحي للأسر بالأخذ بالمبادئ الطبية الأساسية ومعرفتها، وكذا التعرف على قواعد الرعاية الصحية في التغذية الصحية، وتوجيه الناس إلى تحصين أنفسهم من الأمراض من خلال الأحاديث النبوية التي تشي بضرورة تحري النظافة في المسكن والملبس والطعام والمشرب، حيث جعل الإسلام مبدأ أن النظافة من الإيمان من الأسس الاجتماعية التي تقوم عليها معاش الناس والعمل على الحفاظ على أنفسهم ولا عدم الاضرار بالبيئة علاوة على الحفاظ عليها صديقة للمجتمعات لا خصما عليها. إن ضرورة قيام القائمون على الاتصال الجماهيري

15 Ibn Al-Qayyim - altibu alnubuiu- Dar Al Hilal - Beirut - Lebanon, (p. 110). 
أثر الطب النبوي في التوعية الصحية - وباء كورونا أنموذجاً

في استخدام النصوص القرآنية والأحاديث الشريفة في توعية الشباب بحرمة تعاطي

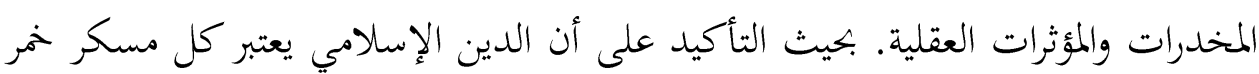

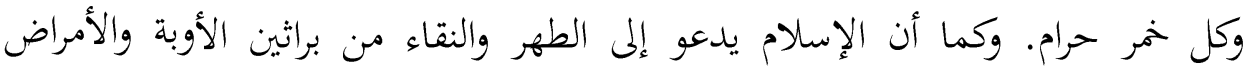
والمخدرات.

إن ما يربط بين المفاهيم العامة لدور الإرشاد الديني ودور وسائل الإعلام في تعزيز الحفاظ

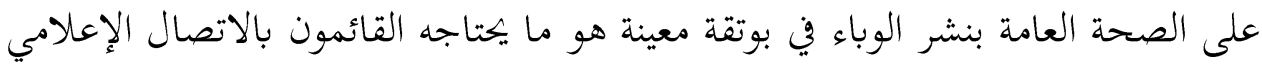

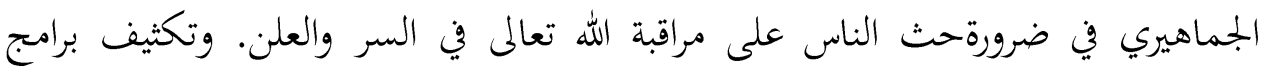

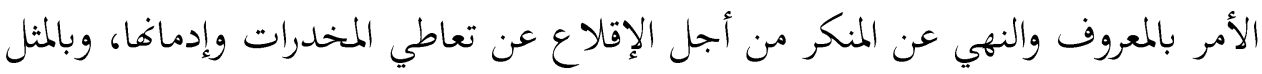

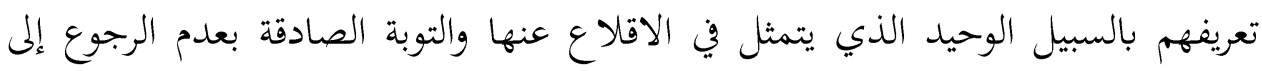
تعاطيها. ولأجل تحقيق هذا المطلب التوعوي الصحي في تعليم المواطنين بمراد الأحاديث النبوية

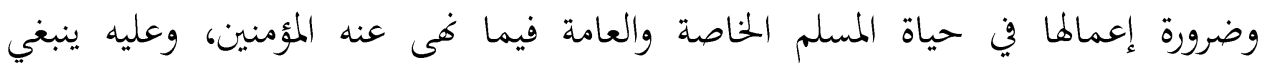

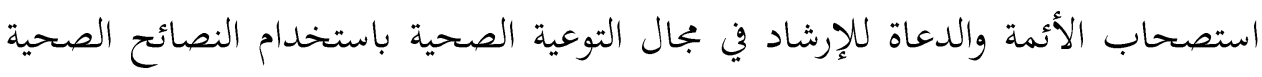

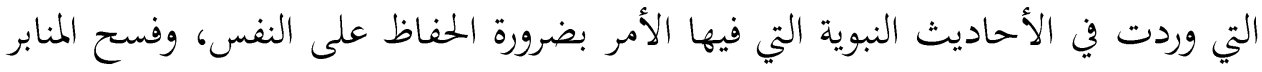

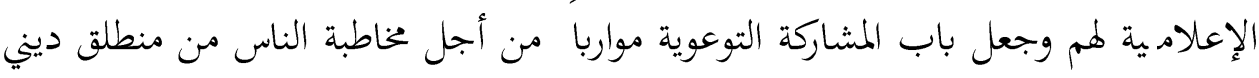

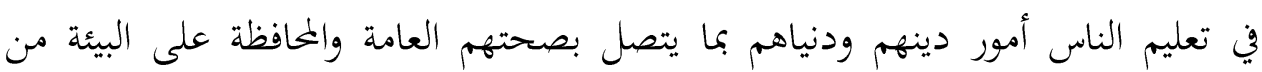

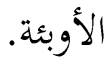
يتضح دور الطب النبويفي التوعية الصحية التي تختص بمكافحة الأوبئة والأمراض في ترسيم

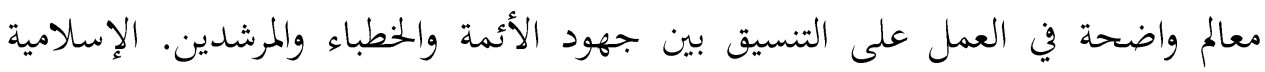
الطوعية والرممية المتمثلة في وزارة الشؤون الإسلامية والدعوة والإرشاد وما يتبع لها من

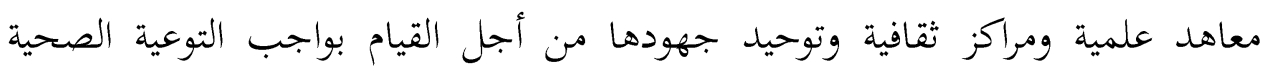

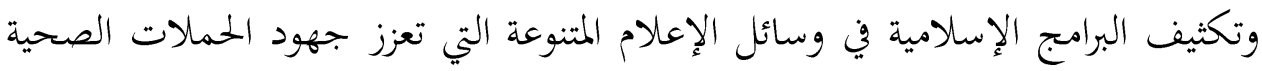

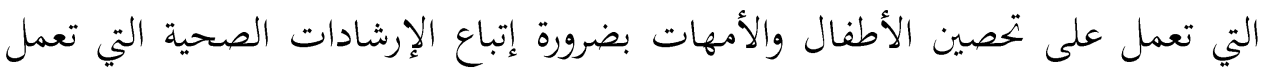
على تحصينهم من الأمراض. 
أثر الطب النبوي في التوعية الصحية - وباء كورونا أنموذجا"

ولا شك أن منبر يوم الجمعة له دور كبير في القيام بمختلف الأنشطة الاجتماعية بجاه إصلاح البيئة الصحية الخاصة بالفرد والمجتمع وهو المدخل المشترك للقائمين بالاتصال الجماهيري للتنسيق بين هذه الجهود وجعلها برنامجا إستراتيجيا تنطلق منه إلى التوعية والتثقيف الصحي بالأوبئة العامة والأمراض الخطيرة. إن المجتمعات الإسلامية تبدو اليوم في أشد الحاجة إلى تكثيف البرامج الدينية في أن تكون يي مقدمة البرامج الإعلامية التوعوية لما تحظى به في نفوس الناس من تعظيم ومن ثم

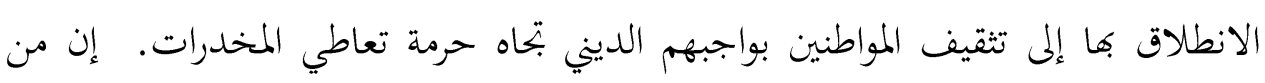
العوامل التي فاقمت من قضية انتشار تعاطي المخدرات الاحباط الكبير الذي يعايشه

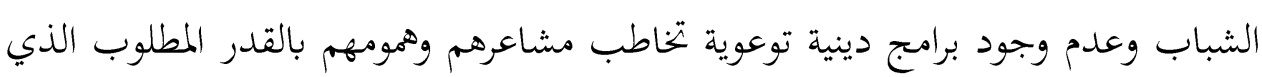
يعمل على اشباع رغباةم الروحية وملئ أوقات فراغهم فيما ينفعهم ولا يضرهم.

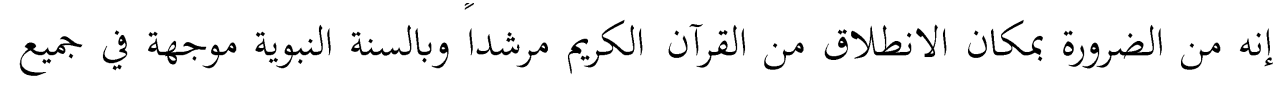
مناحي الحياة خاصة فيما يتصل بالحذر من الأمراض والأسباب المؤدية إليه بخاصة في حالة

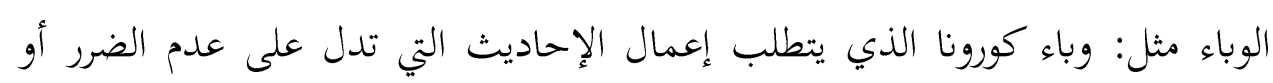
الإضرار بالبيئة الصحية من حوله . ووفقاً لهذا المطلب الشرعي السديد يأتي دور وسائل

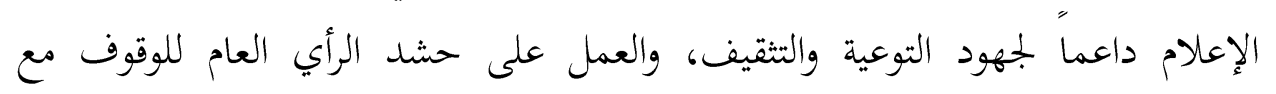
الحملات الإعلامية الرامية إلى الحد من تعاطي الممنوعات الضارة بالصحة العامة، وتوضيح الأدلة الشرعية الرامية إلى الحفاظ على الكليات الخمس. ويكمن دور الطب النبوي أيضا في إظهار مرامي الشرع في الحفاظ على المقاصد الخمسة وهي: الدين، والنفس، والعقل، والعرض، والمال. وبالتأكيد أن فيروس كورونا يعمل على ضياع الدين والنفس والعقلفهو مهلك لهذه الضرورات المثلى التي جاء الشرع في الحفاظ على مكانة النفس وميزة العقل، ولهذا حرمت الأحاديث النبوية الأشربة المسكرة كالخمر والحشيش والأفيون والبنج والدخان على أساس الحفاظ على النفس البشرية وتقويمها من الضياع ومن ذلك ما يصيب المصاب بمرض كورونا من فقدان المناعة الصحية التي تعمل على هلاكك النفس والإضرار بصحة الفرد وضياع النفس والمال. ولما كان العقل مناط التكليف... 


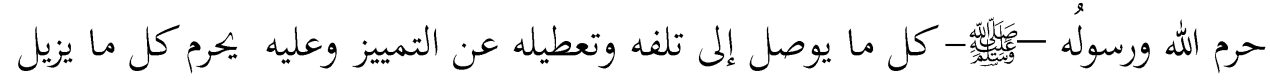

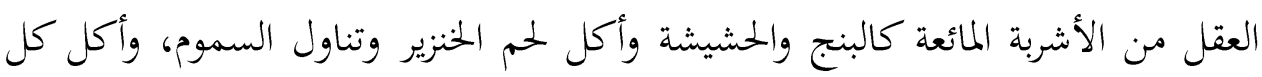

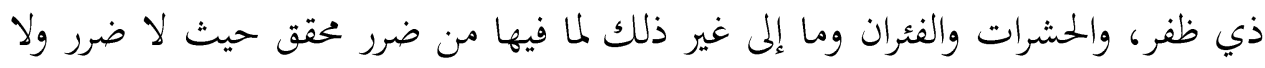

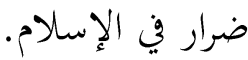
وقد ورد في الأحاديث النبوية البول في المياه، وإهلاك الحرث والنسل وبأن الله لا يحب

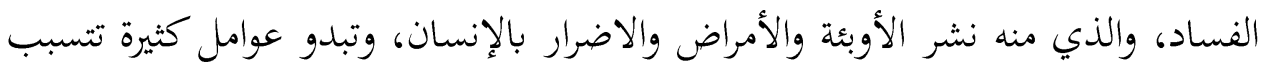

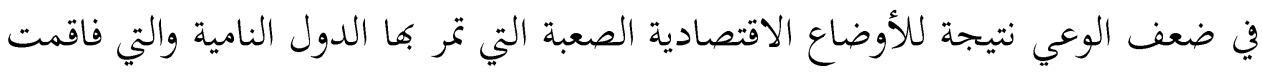

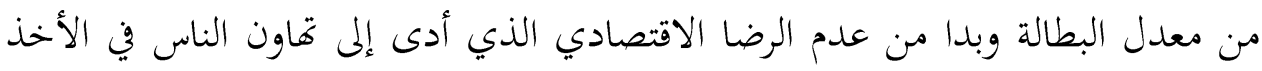

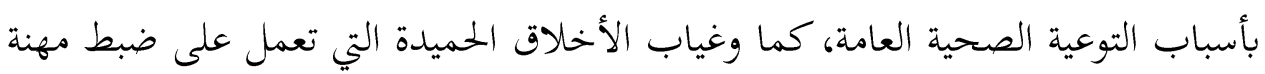
التطبيب، وعدم التلاعب بحياة الناس من نشر الفيروسات والأمراض.

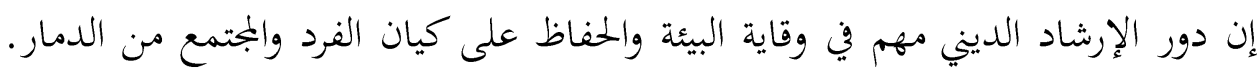

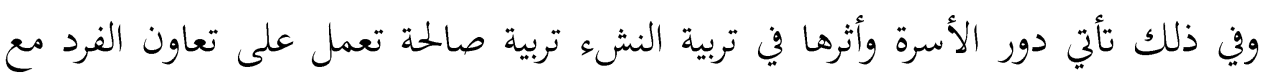

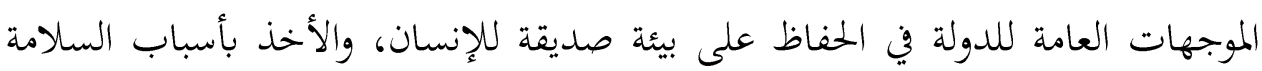

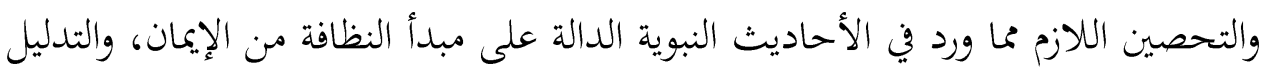

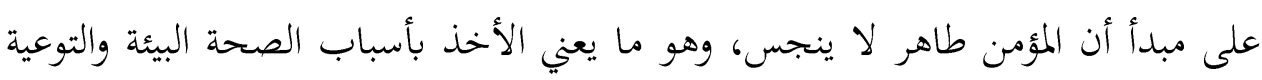

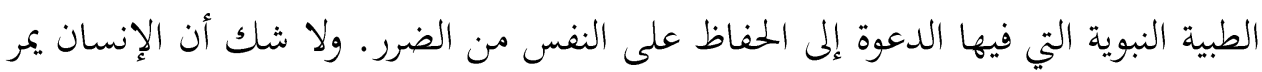

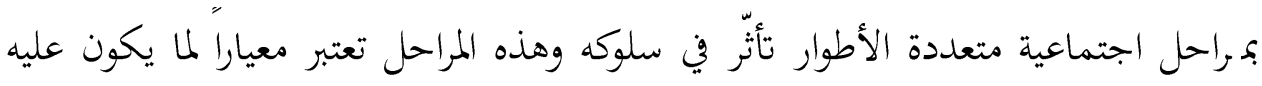

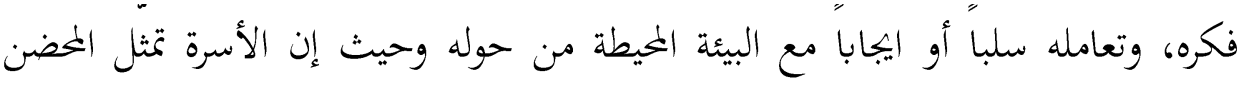

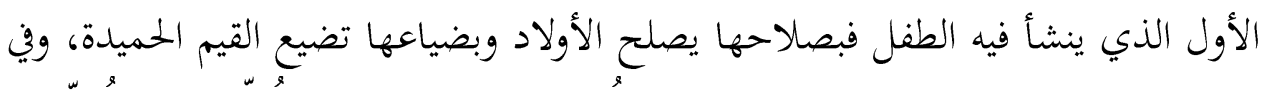

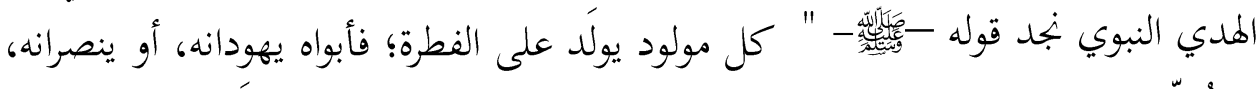
أو يمجسانه" والحديَث دليل على تأثير الأسرة في سلوك الأطفال ويتمثل قمة ذلك في أن ينشأ الأطفال

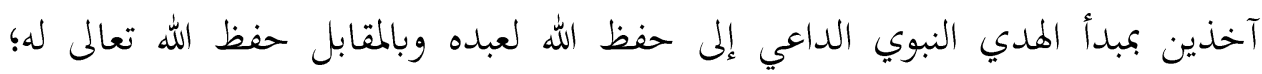

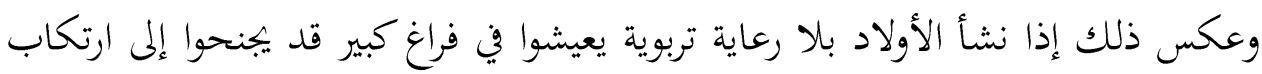


الأفعال المضرة بالصحة والبيئة، فالأسرة من أقوى العوامل التي تسهم في تكوين شخصية

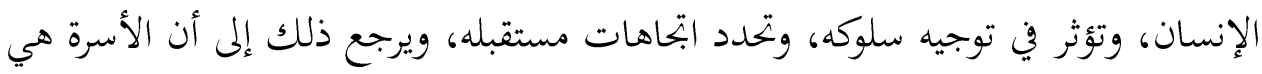

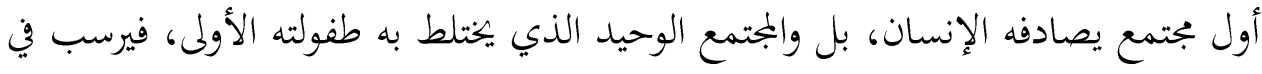

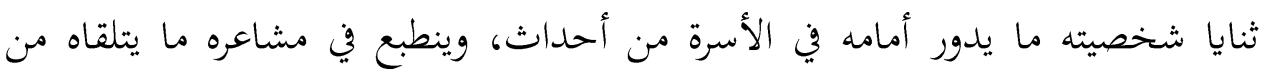
قسوة أو ورقة، وعناية أو إهمال. يتضمن دور وسائل الإعلام استهداف الأسر بالبرامج التوعوية المستديمة وتعريفها بضرورة التمسك بالأخلاق الفاضلة التي تدعو إلى النظافة والمحافظة على البيئة، وعدم الاضرار

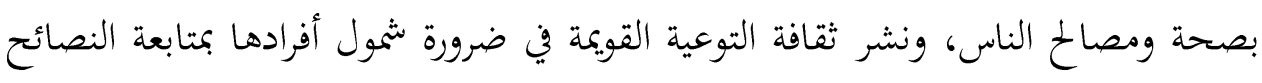

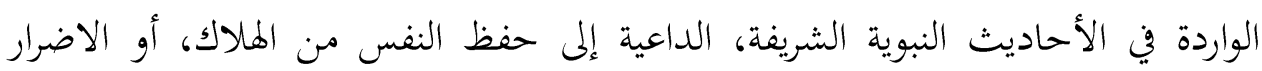
بالآخرين مثل البيئة والمجتمع. يرتكز دور العمل بمقاصد الشرع في التوعية الصحية الواردة في الأحاديث النبوية بأخما تعمل

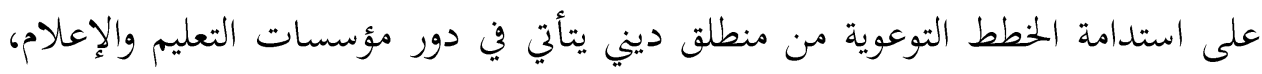

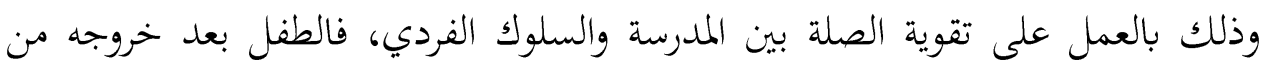

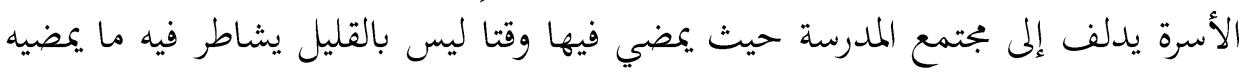

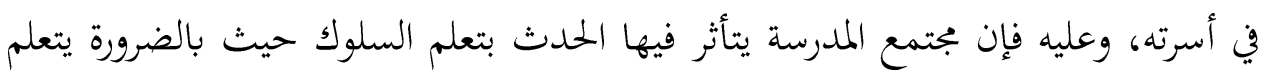

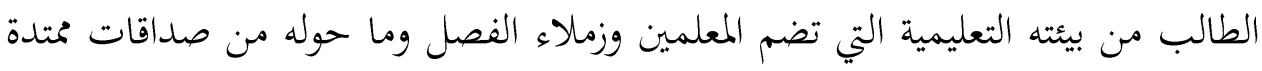

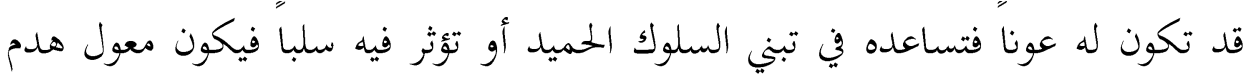

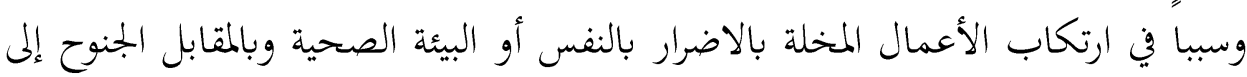

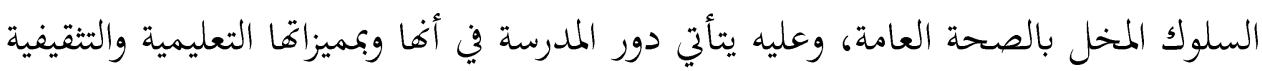

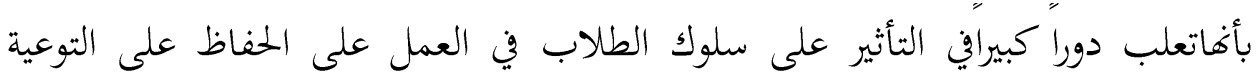

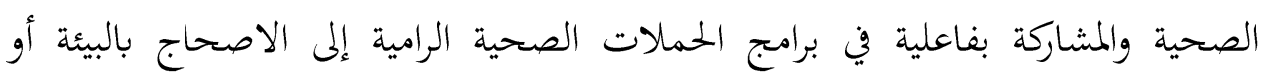

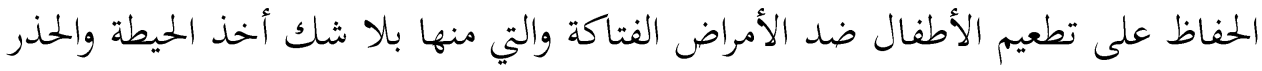

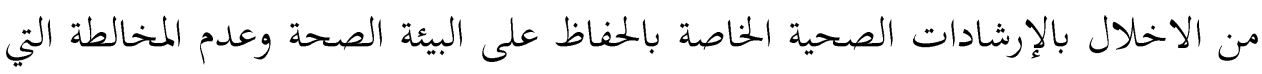

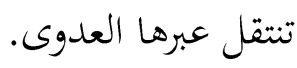


إن دور الطب النبوي في التوعية الصحية يكمن في مكافحة العادات الصحية السالبة التي تعمل على نشر وباء كورونا من خلال السلوك الصحي المشين المتمثل في عدم الانضباط بالقواعد الصحية المتبعة في المحافظة على المعاشرة الاجتماعية بعيدا عن الاختلاط أو المصافحة أو المشاركات في الأنشطة الاجتماعية التي يكتز فيها المشاركون من دون لبس الكمامات أو إتباع قواعد التباعد الاجتماعي. وعليه المطلوب من العاملين في مجال الإعلام المختص بالتوعية الصحية ترتيب الأولويات بتركيز برامج التوعية الصحية من أجل استهداف شرائح اجتماعية مختلفة بخاصة فئة الشباب من غختلف الأعمار بالتثقيف المستدام، وصبغ البيئة التعليمية ببرامج الإرشاد التربوي الصحي والاجتماعي والنفسي، ويتعاظم ذلك الدور إذا ما علم أن التقارير الرسمية والشبه رسمية تؤكد ازدياد تعاطي المخدرات بين طلاب المدارس والجامعات. إن دور الإرشاد الديني يعد من أولى الأولويات لما له من تأثير كبير على المجتمعات الإسلامية، وعليه فمخاطبة الناس بأهمية مراعاة التوجيهات النبوية بمقصد الشرع الحكيم في تحريمه الاضطرار البيئة وتلويثها حيث يعد هذا الخطاب من أهم المداخل القوية التي تعمل على الاستجابة لضبط الحياة الاجتماعية على أسس صحية قويمة توفر العيش الكريم وتهيئة البيئة وجعلها صالحة للعيش بصحة وأمان. وبذا يتوجب على الأئمة الدينيين والقائمين بالاتصال الجماهيري ومؤسسات المجتمع المني الاستفادة من المنابر الدينية بالتنسيق مع أئمة المساجد ومديري البرامج الدينية في كافة وسائل الإعلام من أجل مخاطبة المواطنين وخاصة فئة الشباب بضرورة الانخراط في جهود التوعية الصحية التي تتطلب التوعية والتثيف في تفعيل التوجيهات الصحية التي وردت في الأحاديث النبوية وجعلها مرشدا للمعاشرة الاجتماعية بمختلف نواحيها.

خاتمة هذه الورقة العلمية (أثر الطب النبوي في التوعية الصحية) تكمن أهميتها في الحفاظ على النفس البشرية وصحة البيئة من خلال تطبيق مبدأ النظافة من الإيمان وعدم الاضرار 
كلياة الناس استنادا على مبدأ لا ضرر ولا ضرار. وعليه فيمكن إجمال نتائج البحث فيما

- - ميعز الطب النبوي حرمة قتل النفس البشرية

- - يطبق الطب النبوي مقصد المحافظة على النفس

- - الإسلام دين متكامل شامل لكل مناحي الحياة بخاصة الصحية

- - الطب النبوي يقوم على مبدأ لا ضرر ولا ضرار

- م الطب النبوي يطبق مبدأ النظافة من الإيمان

- الطب النبوي يوجه إلى محاصرة الأوبئة والأمراض في أماكنها. - - منابر الجمعة هي من أهم الوسائل التوعية في الثثقيف بالتوعية الصحية

أهم توصيات البحث

- - - ل ضرورة انطلاق الحملات التوعوية من المنابر الإسلامية والتي من أهما المساجد

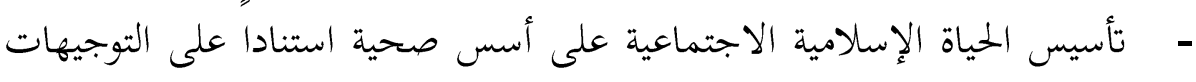

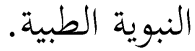

$$
\begin{aligned}
& \text { - - اتخاذ شعار لا ضرر ولا ضرار في الحملات التوعوية الصحية } \\
& \text { - ادخال مادة باسم الطب النبوي ضمن مقررات المراحل الدراسية } \\
& \text { - التشجيع على البحوث الصحية القائمة على توجيهات الطب النبوي. }
\end{aligned}
$$

\section{(c) (1) (2)}

BY NC SA This work is licensed under a Creative Commons Attribution-NonCommercial-ShareAlike 4.0 International (CC BY-NC-SA 4.0) 